\title{
Safety of Recreational Water Slides: Numerical Estimation of the Trajectory, Velocities and Accelerations of Motion of the Users
}

\author{
Piotr Szczepaniak and Ryszard Walentyński \\ Silesian University of Technology, \\ Faculty of Civil Engineering, \\ ul. Akademicka 5, PL44-100 Gliwice, Poland \\ Piotr.Szczepaniak@polsl.pl, Ryszard.Walentynski@polsl.pl \\ http://www.kateko.rb.polsl.pl
}

\begin{abstract}
The article briefly shows how to estimate the safety of recreational water slides by numerical analysis of motion of the users. There are presented: mathematical description of a typical water slides geometry, simplified model of a sliding person, model of contact between the user and the inner surface of the slide, equations of motion written for a rigid body with 6 degrees of freedom and finally some sample results compared to the limitations set by current European Standard.
\end{abstract}

Keywords: water slide, safety, water park, motion, dynamics, finite difference, numerical integration, modeling, Mathematica.

\section{Introduction}

Water slides are one of the most popular facilities in water parks. They are built all over the world. One of the most important problems associated with them is safety. It is not well recognized, both mathematically and technically. There are very few scientific papers concerning the problem. The most complete publication [1] deals with the mathematical model of a water sliding process using the assumption that the user has constant contact with the inner surface of the chute. Actually, the most dangerous situations happen when the user looses contact and cannot control the ride. The next problem is acceleration, often called G-load. The human body, and especially brain and heart, is sensitive to acceleration that significantly exceeds gravity acceleration $g$. Several accidents, in a variety of countries resulted in severe injuries and even death.

The contemporary practice, due to lack of design methodology, consists in testing by a water slides expert after finishing the construction, however this expert is described only as a fit person, dressed in bathing suit [2]. It is not acceptable from the point of view of modern engineering philosophy, which requires prior analysis based on mathematical model, which should be verified experimentally. 


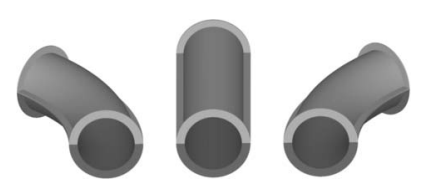

Fig. 1. Basic elements of water slides

\section{Typical Geometry of Water Slides}

Most of the water slides (especially those built high over the ground level) are constructed of two basic types of elements: the straight ones, being just a simple cylinder, and the curved ones, having the shape of a slice of a torus. All these elements are equipped on both ends with flanges that allow connecting the following parts of the slide with screws (see Fig. 11 and 2). Because the resulting axis of the chute consists of straight lines and circular arcs, it can't be described by a simple equation, but some kind of an interval changing function is needed.

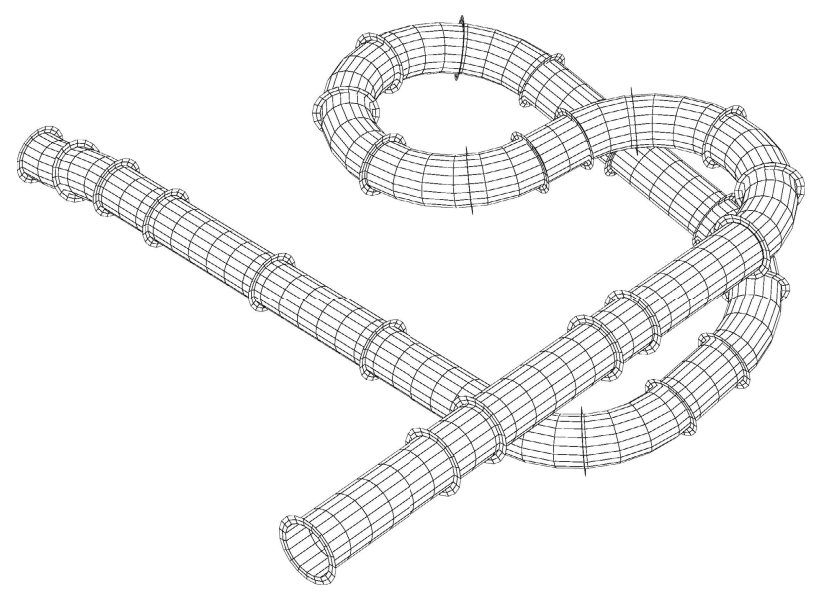

Fig. 2. A sample shape of a chute

The best choice seems to be the IntepolatingFunction within the Mathematica system. To obtain a parametric equation of the axis, one must first calculate a set of coordinates of discrete points, lying on the axis at small intervals. It can be done using procedures described in 3] or with the aid of any software for 3-D graphics. The next step is to build an IntepolatingFunction for each of the coordinates separately and finally join them into one vector function axis [1]. Having this function one can get the parametric equation of the surface of the slide surface[1,phi,radius] using the following Mathematica code: 


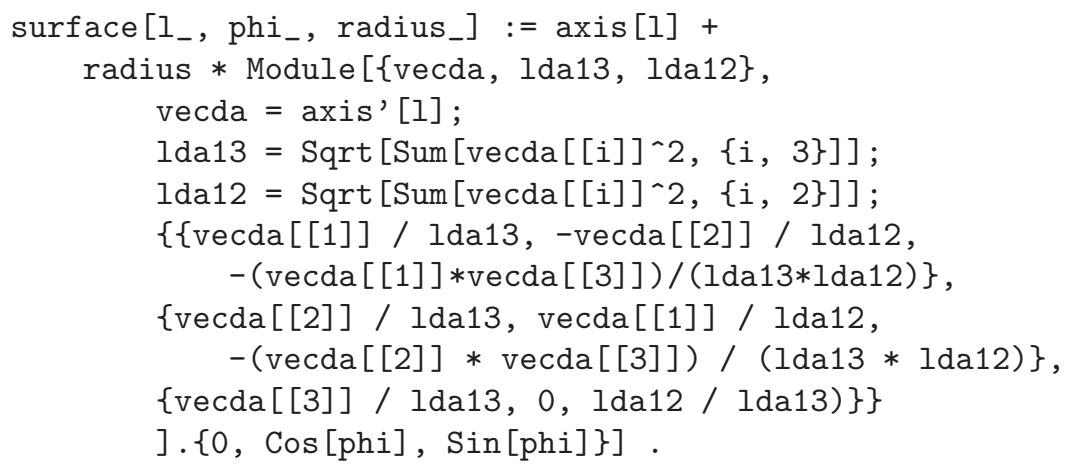

Within the above code 1 denotes the position of a current cross-section, measured along the axis, phi and radius are cylindrical coordinates of points creating the cross-section. Figure 2 has been created with this code and the ParametricPlot3D command.

\section{Model of a Sliding Person}

The next task is to create a model of the human body. It's obvious, that a complete bio-mechanical model with all its degrees of freedom (DOF) and parameters would be the best, but unfortunately it's almost impossible to predict the values of dimensions, mass, moments of inertia and stiffness of all parts of the users' body, especially at the design stage of the construction process. That's why a simpler model is needed.

To create it, one can notice, that sliding people are quite stiffened and the fastest users touch the surface of the chute only with their heels and bladebones. This allows us to replace the sliding person by a rigid body, constrained by 3 unilateral supports located at the vicinity of the previous mentioned parts of the body (spheres at the vertices of the triangle representing the body on Fig. 3). Such a body has 6 DOFs - translations $x_{i}$ and rotations $\theta_{i}$ around 3 axes of the local $\left(\xi_{i}\right)$ or global $\left(\mathrm{X}_{i}\right)$ coordinates system, and is subjected to the influence of the following forces: gravity $\boldsymbol{F}_{\mathrm{G}}$, normal contact forces $\boldsymbol{F}_{\mathrm{N}_{i}}$ and friction forces $\boldsymbol{F}_{\mathrm{T}_{i}}$, shown on Fig. 4 . Vectors of these forces can be calculated using the following formulae:

$$
\begin{gathered}
\boldsymbol{F}_{\mathrm{N}_{i}}=\left\{\begin{array}{l}
\mathbf{0} \text { if }\left(u_{i}<0\right) \vee\left[\left(k u_{i}+c u_{i} \dot{u}_{i}\right)<0\right] \\
\left(k u_{i}+c u_{i} \dot{u}_{i}\right) \boldsymbol{n}_{\boldsymbol{i}} \text { otherwise }
\end{array}\right. \\
\boldsymbol{F}_{\mathrm{T}_{i}}=-\mu\left|\boldsymbol{F}_{\mathrm{N}_{i}}\right| \frac{\boldsymbol{v}_{\mathrm{T}_{i}}}{\left|\boldsymbol{v}_{\mathrm{T}_{i}}\right|} \\
\boldsymbol{F}_{\mathrm{G}}=m \boldsymbol{g} \\
\boldsymbol{F}_{\mathrm{Sum}}=\sum_{i=1}^{3}\left(\boldsymbol{F}_{\mathrm{N}_{i}}+\boldsymbol{F}_{\mathrm{T}_{i}}\right)+\boldsymbol{F}_{\mathrm{G}}
\end{gathered}
$$




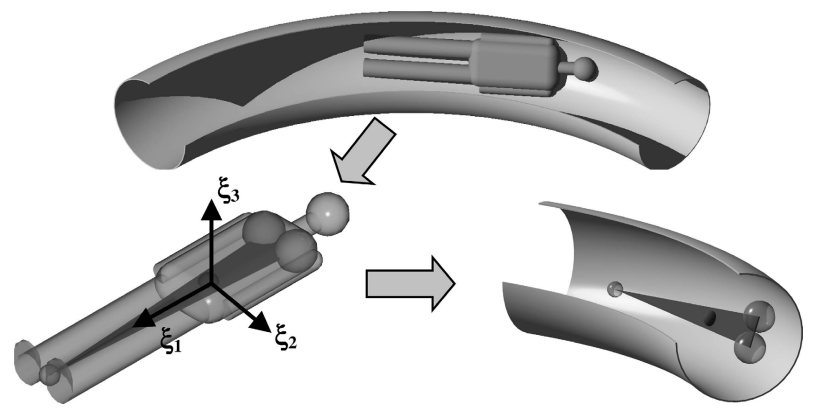

Fig. 3. Replacement of a user by a model of a rigid body

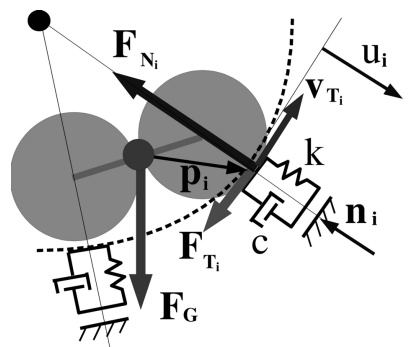

Fig. 4. Model of contact between the moving body (grey circles) and the inner surface of the chute (dotted line). Dot (black) in the upper left corner denotes the center of the current cross-section (axis of the slide).

$$
\boldsymbol{M}_{\mathrm{Sum}}=\sum_{i=1}^{3} \boldsymbol{p}_{i} \times\left(\boldsymbol{F}_{\mathrm{N}_{i}}+\boldsymbol{F}_{\mathrm{T}_{i}}\right)
$$

where:

$i$ - number of the current zone of contact,

$k, c-$ constants of the quasi viscous-elastic model of human body,

$u_{i}$ - "deflection" of the zone of contact,

$\boldsymbol{n}_{i}$ - unit vector, normal to the surface of the slide,

$\mu-$ coefficient of friction,

$\boldsymbol{v}_{\mathrm{T}_{i}}$ - tangent component of the velocity vector,

$m-$ mass of the user,

$\boldsymbol{g}$ - vector of gravitational acceleration,

$\boldsymbol{F}_{\text {Sum }}$ - summary force,

$\boldsymbol{M}_{\text {Sum }}$ - summary moment of forces.

At this stage the main problem is calculating $u_{i}$ and $\boldsymbol{n}_{\boldsymbol{i}}$. The best way to solve it, is to find on the axis of the slide these points, that are nearest to the current 
positions of the centers of the contact spheres, because vectors $\boldsymbol{n}_{\boldsymbol{i}}$ must lie on lines connecting these pairs of points. It can be easily done with the Mathematica FindMinimum command.

\section{Equations of Motion}

The applied equations of motion are based on the well known Newton's laws of motion [4]:

$$
\begin{aligned}
& \frac{d^{2}}{d t^{2}}[m \boldsymbol{x}(t)]=\boldsymbol{F}_{\mathrm{Sum}}, \\
& \frac{d}{d t}[\boldsymbol{K}(t)]=\boldsymbol{M}_{\mathrm{Sum}}, \\
& \boldsymbol{K}(t)=\boldsymbol{A}(t) \cdot \boldsymbol{J} \cdot \boldsymbol{\omega}(t), \\
& \boldsymbol{A}(t)=\left(\begin{array}{ccc}
\cos \left[\theta_{3}(t)\right] & -\sin \left[\theta_{3}(t)\right] & 0 \\
\sin \left[\theta_{3}(t)\right] & \cos \left[\theta_{3}(t)\right] & 0 \\
0 & 0 & 1
\end{array}\right) \cdot\left(\begin{array}{ccc}
\cos \left[\theta_{2}(t)\right] & 0 \sin \left[\theta_{2}(t)\right] \\
0 & 1 & 0 \\
-\sin \left[\theta_{2}(t)\right] & 0 \cos \left[\theta_{2}(t)\right]
\end{array}\right) \\
& \cdot\left(\begin{array}{ccc}
1 & 0 & 0 \\
0 & \cos \left[\theta_{1}(t)\right] & -\sin \left[\theta_{1}(t)\right] \\
0 & \sin \left[\theta_{1}(t)\right] & \cos \left[\theta_{1}(t)\right]
\end{array}\right), \\
& \boldsymbol{J}=\left(\begin{array}{ccc}
J_{\xi 1} & 0 & 0 \\
0 & J_{\xi 2} & 0 \\
0 & 0 & J_{\xi 3}
\end{array}\right) \\
& \boldsymbol{\omega}(t)=\frac{d \theta_{1}(t)}{d t} \cdot\left(\begin{array}{l}
1 \\
0 \\
0
\end{array}\right)+\frac{d \theta_{2}(t)}{d t} \cdot\left(\begin{array}{c}
0 \\
\cos \left[\theta_{1}(t)\right] \\
-\sin \left[\theta_{1}(t)\right]
\end{array}\right)+ \\
& +\frac{d \theta_{3}(t)}{d t} \cdot\left(\begin{array}{c}
-\sin \left[\theta_{2}(t)\right] \\
\sin \left[\theta_{1}(t)\right] \cos \left[\theta_{2}(t)\right] \\
\cos \left[\theta_{1}(t)\right] \cos \left[\theta_{2}(t)\right]
\end{array}\right)
\end{aligned}
$$

where:

$$
\begin{aligned}
\boldsymbol{K}(t) & - \text { vector of moment of momentum, } \\
\boldsymbol{A}(t) & \text { - matrix of transformation from local (rotating) } \\
& \text { to global (fixed) coordinates system, } \\
\boldsymbol{J} & - \text { tensor of main moments of inertia, } \\
\boldsymbol{\omega}(t) & \text { - vector of angular velocity. }
\end{aligned}
$$

As one can see, the equations of motion are so complicated, that it is impossible to obtain an analytical solution. In fact, even NDSolve, the numerical solution of differential equations in Mathematica, does not work due to the usage of the FindMinimum command within the equations of motion (see Sect. 33). So a special code had to be written, and it follows an algorithm based on a combination of the Taylor's series and multi-step methods [5]: 


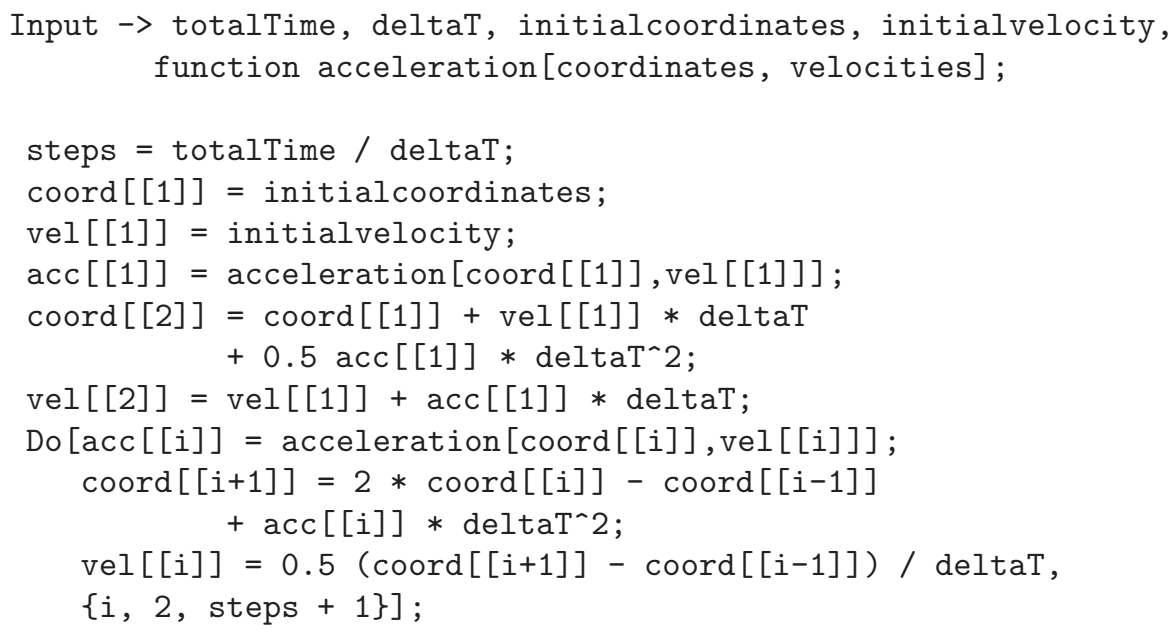

Output $\rightarrow$ lists: $\operatorname{coord}[[i]], \operatorname{vel}[[i]], \operatorname{acc}[[i]]$.

Of course, each single element of the output lists coord[[i]], vel[[i]] and $\operatorname{acc}[[i]]$ is a 6 dimensional vector. This code seems to be stable and a sufficient precision is reached with deltaT $=0.002$ [second].

\section{Sample Results}

Some sample results were obtained for the slide shown in Fig. 2, There is shown the trajectory of the motion and the axis of the slide in Fig. 5. The beginning is at the point $(0,0)$, and then the body goes along the direction of the $\mathrm{X}_{2}$ axis, next turns left and follows the curved axis of the slide, slightly swinging. More

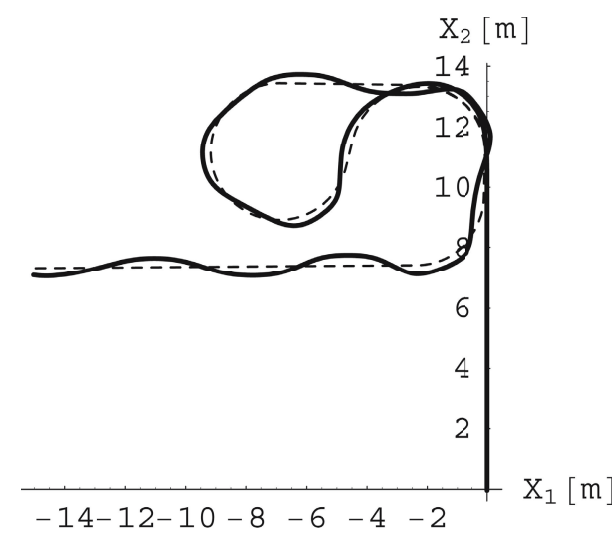

Fig. 5. Top view of the axis of the slide (dotted line) and the trajectory of motion (bold line) 

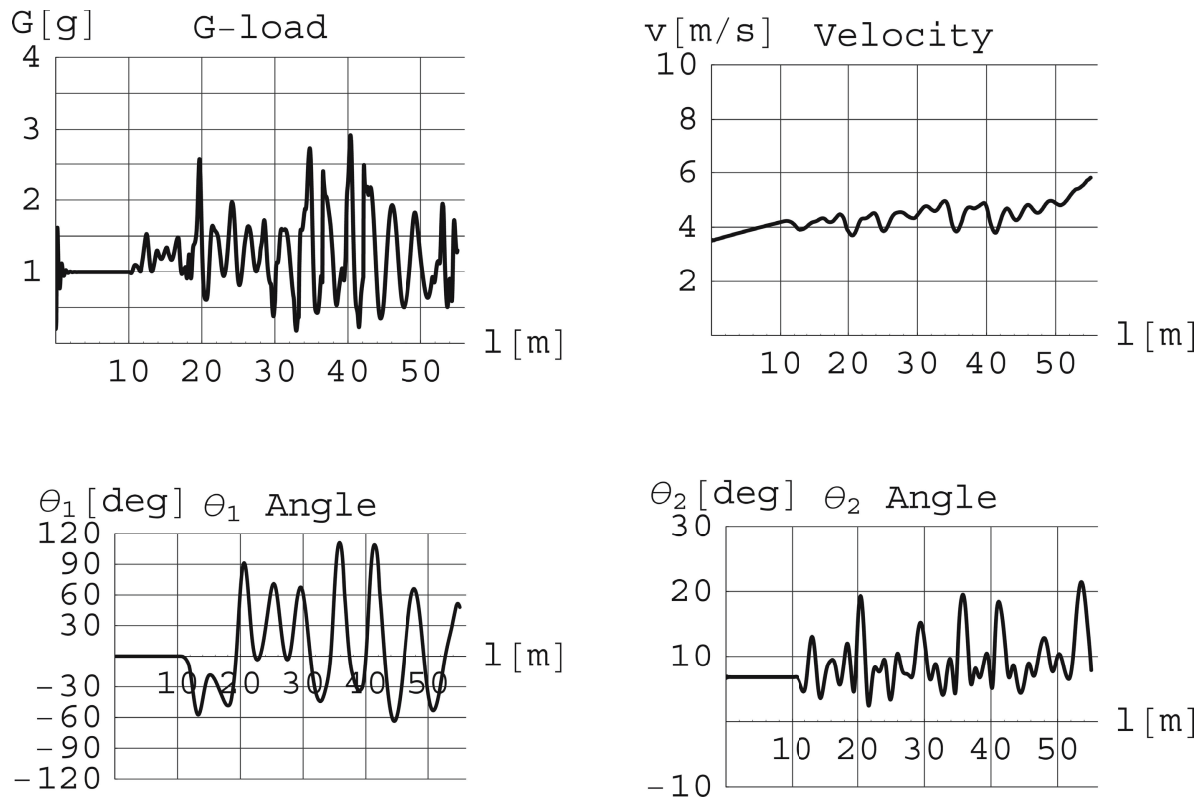

Fig. 6. Sample results (description in text)

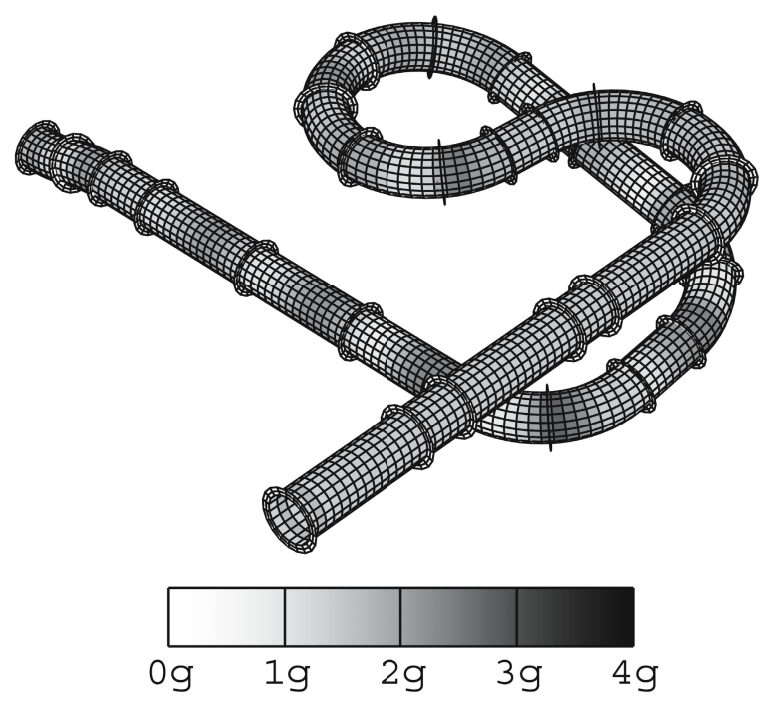

Fig. 7. Results of the G-load calculations. Shades of grey denotes different values of $G$.

detailed results can be read from Fig. 6. There are presented: the G-load acting on the moving body

$$
G=\left|\boldsymbol{F}_{\mathrm{Sum}}-\boldsymbol{F}_{\mathrm{G}}\right| /(9.81 \cdot m)
$$


value of velocity and first and second angular coordinates (longitudinal and transversal rotations around $\xi_{1}$ and $\xi_{2}$ axes - see Fig. 3), where on the horizontal axes of these charts is marked the length of the chute.

The current design code [2] sets some limitations on the values of permissible G-load for safety reasons. It says, that $G \leq 2.6 \mathrm{~g}$ is safe, and $2.6 \mathrm{~g}<G \leq 4.0 \mathrm{~g}$ is acceptable, but only for less then 0.1 second. Within the presented example these limitations are kept, but sometimes it is hard to do so. Especially the second condition can cause some problems when designing a very steep and fast water slide, where the high speed generates huge centrifugal force at each bend.

\section{Conclusions}

Numerical modelling of motion is a good method of checking the geometry of water slides during the design process. It allows to estimate the excitement level of the ride, which is dependent on speed and acceleration, and points out the dangerous parts of the slide, where the value of G-load is to high (exceeds 2.6 or $4.0 \mathrm{~g}$ ). The applied algorithms are user-friendly, and the results of computations can be presented in many forms, beginning from pure numbers and ending on pictures like Fig. 7 and animations of the motion.

Acknowledgments. This paper has been supported by the Polish Committee of Scientific Research (grant No. 0416/T02/2006/31).

\section{References}

1. Joo, S.-H., Chang, K.-H.: Design for the safety of recreational water slides. Mech. Struct. \& Mach. 29 (2001) 261-294

2. European Standard EN 1069-1:2000 Water slides of $2 \mathrm{~m}$ height and more - Part 1: Safety requirements and test methods. CEN, Bruxelles (2000)

3. Szczepaniak, P.: Zjeżdżalnie wodne. Obliczanie geometrii zjeżdżalni i modelowanie ruchu użytkownika (Water slides. Calculating the geometry and modelling of a motion of a user). MSc thesis. Silesian University of Technology, Gliwice (2003)

4. Borkowski, Sz.: Mechanika ogólna. Dynamika Newtonowska (General Mechanics. Newton's Dynamics). 2nd edn. Silesian University of Technology Press, Gliwice (1995)

5. Burden, R.L., Faires, J.D.: Numerical Analysis. 5th ed. PWS Publishing Company, Boston (1993) 